\title{
Objetivos de seleção e valores econômicos em sistemas de produção de gado de corte no Brasil ${ }^{1}$
}

\author{
João Jorge Júnior ${ }^{2}$, Vera Lucia Cardoso ${ }^{3}$, Lucia Galvão de Albuquerque ${ }^{4}$ \\ ${ }^{1}$ Auxílio financeiro: $C N P q-F A P E S P$. \\ 2 Doutorando em Produção Animal - FCAV - UNESP - Jaboticabal. \\ ${ }^{3}$ APTA Regional/SAA-SP, PRDTA Centro Leste, Ribeirão Preto, SP. \\ ${ }^{4}$ Departamento de Zootecnia - FCAV - UNESP - Jaboticabal. Pesquisadora do CNPq.
}

RESUMO - A importância econômica de possíveis características biológicas a serem incluídas em objetivos de seleção para diferentes sistemas de produção de bovinos da raça Nelore, mediante o cálculo dos seus valores econômicos foi avaliada nesta pesquisa. Com base em informações de desempenho e parâmetros biológicos e econômicos, foram simulados diferentes sistemas de produção (ciclos de cria e completo) para dois rebanhos. O rebanho 1, com ciclo de cria (Ccr), ciclo completo (Cco) e ciclo completo com venda de reprodutores (CcoR), é um rebanho elite no qual é desenvolvido um programa seleção. Parte deste rebanho é também destinada à produção de animais comerciais. O rebanho 2 é um rebanho exclusivamente comercial, com Ccr e Cco. Os valores econômicos foram calculados usando-se um modelo bio-econômico, para as seguintes características: peso (PD) e taxa de desmama (TD), peso da vaca adulta (PVA), ganho médio diário no confinamento (GMD), pesos ao abate (PA) e de carcaça (PC), peso final dos tourinhos (PFT), rendimento de carcaça (RC) e consumo alimentar no confinamento (CAc) e em pastagem (CAp). Para os sistemas de ciclo completo e de ciclo completo com venda de reprodutores e CcoR (Cco e CcoR), os valores econômicos variaram de R\$ 0,34 a R \$ 0,40 para PD, R \$ 3,51 a R \$ 10,15 para TD, -R\$ 0,16 a R\$ 0,09 para PAV, R\$ 0,32 a R\$ 0,76 para GMDc, R\$ 1,09 a R\$ 1,17 para PA; R \$ 2,03 a R\$2,19 para PC, R\$ 23,89 a R\$28,61 para $\mathrm{RC}$, e R\$11,85 para PFT, - R\$0,45 para CAc e - R \$ 0,03 para CAp. A taxa de desmama e o rendimento de carcaça foram as características de maior impacto no lucro anual dos dois rebanhos. As análises de sensibilidade demonstraram que, de modo geral, possíveis mudanças nos preços de insumos e produtos influenciariam de forma mais significativa os valores econômicos nos sistemas de produção nos quais esses preços eram mais elevados nas situações básicas.

Palavras-chave: análise de sensibilidade, ciclo completo, ciclo de cria, manejo alimentar, Nelore

\section{Breeding goals and economic values for beef cattle production systems in Brazil}

\begin{abstract}
Economic values were calculated to evaluate the economic importance of possible traits to be included in breeding goals, for different production systems of Nelore cattle. Two types of production systems (cow-calf and complete breeding cycles) were simulated for two types of herds. Herd 1 was defined as a nucleus herd (where selection was carried out and a percentage of animals were also raised for commercial purposes), with cow-calf $(\mathrm{Cc})$, complete breeding cycle ( $\mathrm{Cbc}$ ) and complete breeding cycle with sales of animals for reproduction (CbcR). Herd 2 was defined as an exclusively commercial herd, with cow-calf cycle (Cc) and complete breeding cycle $(\mathrm{Cbc})$. Using a bio-economic model, economic values were calculated for the following traits: weaning weight (WW), weaning rate (WR), mature weight (MW), average daily gain in feed-lot (DAG), slaughter (WS) and carcass weights (WC), young bulls' final weight (FWYB), dressing percentage (DP), feed-lot (FLFI) and pasture feed intakes (PFI). The economic values for $\mathrm{Cbc}$ and $\mathrm{CbcR}$ (herds 1 and 2) ranged from $\mathrm{R} \$ 0.34$ to $\mathrm{R} \$ 0.40$ for $\mathrm{WW}, \mathrm{R} \$ 3.51$ to $\mathrm{R} \$ 10.15$ for $\mathrm{WR},-\mathrm{R} \$ 0.16$ to $\mathrm{R} \$ 0.09$ for MW, R\$ 0.32 to $\mathrm{R} \$ 0.76$ for FLFI, R\$ 1.09 to $\mathrm{R} \$ 1.17$ for WS; R\$2.03 to R\$2.19 for WC, $\mathrm{R} \$ 23.89$ to $\mathrm{R} \$ 28.61$ for $\mathrm{YG}$, $\mathrm{R} \$ 11.85$ for FWYB, - R \$ 0.45 for FLFI and - R \$ 0.03 for PFI. WR and DP presented the largest impacts on annual profits in both herds. Sensitivity analyses indicated that, in general, possible changes in prices of production components and products would exert a greater influence on the economic values for those production systems with higher prices in the basic situation.
\end{abstract}

Key Words: complete breeding cycle, cow-calf cycle, feed management, Nelore, sensitivity analysis

\section{Introdução}

Nos últimos anos, os trabalhos nos programas internacionais de melhoramento têm sido direcionados no sentido de obter animais com maiores eficiências produtiva e reprodutiva, mantendo um balanço adequado entre características de produção, fertilidade e adaptabilidade. Um passo muito importante para esta melhora é o estabele- 
cimento de objetivos de seleção, que podem ser definidos como a combinação dos valores genéticos das características economicamente importantes em determinado sistema de produção, ponderados por seus respectivos valores econômicos relativos (Ponzoni \& Newman, 1989). Atualmente, no Brasil, existem alguns programas de melhoramento que utilizam índices empíricos. Essas avaliações foram implantadas sem um estudo específico para definição dos objetivos de seleção em termos econômicos. É fundamental o trabalho que esses grupos têm realizado nesses últimos anos, porém, há poucos estudos referentes à otimização do uso combinado de DEP (diferenças esperadas na progênie) para maximizar a resposta à seleção.

Uma clara definição dos objetivos de seleção é muito importante para o desenvolvimento de estratégias de melhoramento genético que sejam custo-efetivas e identificação de critérios de seleção que contribuam na predição acurada das características que afetam a lucratividade de rebanhos comerciais (Amer et al., 2001). A partir da definição dos objetivos de seleção, são escolhidas as características, ou critérios de seleção, que serão utilizadas na predição dos valores genéticos dos indivíduos. As características prédeterminadas são a base para formulação da função de lucro a partir da qual são derivados os valores econômicos (Parnell, 2000).

Em uma propriedade, várias características biológicas afetam as receitas e os custos. Essas características podem ser separadas em quatro grupos: de crescimento, reprodutivas, de ingestão de alimento e de carcaça.

A importância econômica dessas características biológicas a serem incluídas em um objetivo de seleção é aferida pelos seus valores econômicos (VE), definidos como o au mento esperado no lucro anual do rebanho resultante do aumento em uma unidade de uma característica (supondo que as demais sejam mantidas constantes), em decorrência de seleção. Valores econômicos incorretos ou a omissão de características importantes pode ocasionar perda de eficiência no melhoramento da produção animal (Groen et al. 1997).

Objetivou-se neste trabalho estudar as características a serem incluídas em programas de seleção e obter valores econômicos para diferentes rebanhos de gado de corte, definidos com base no manejo alimentar.

\section{Material e Métodos}

Foram calculados valores econômicos para dois tipos de rebanho, definidos com base no manejo alimentar, considerando, para cada um, tanto o ciclo de cria como ociclo completo. O rebanho 1 (com três ciclos) refere-se a um rebanho mantido em pastagens de braquiária, com suplementação volumosa (silagem de sorgo) e concentrada (farelo de sorgo, levedura de cana e uréia) na seca. O rebanho 2 (com dois ciclos) é um rebanho comercial mantido em pastagens durante o ano todo sem nenhum tipo de suplementação.

As informações de parâmetros biológicos e econômicos utilizados para descrever o rebanho de ciclo completo são provenientes de uma propriedade localizada na região noroeste do estado de São Paulo, que possui um rebanho de 10.500 matrizes, distribuídas em aproximadamente 8.000 ha de pastagens, formadas em sua maioria por braquiárias. A especificação do sistema de produção e de mercado com a descrição do manejo e da alimentação, a composição do rebanho, a idade e o destino dos animais nas diferentes categorias pode ser obtida em Jorge Jr. et al. (2006). Com base nas informações originais, foram simulados três sistemas de produção (Tabela 1) para o rebanho 1: ciclo de cria (Ccr) - quase a totalidade dos bezerros é vendida à desmama, restando na propriedade apenas animais para reposição; ciclo completo (Cco) - todos os machos são destinados ao abate; ciclo completo com venda de reprodutores (CcoR) metade dos machos é destinada ao abate e a outra metade é comercializada como reprodutores.

Para descrever o rebanho comercial (rebanho 2), foram utilizados índices zootécnicos de um rebanho comercial azebuado de corte mantido sob estratégias adequadas de manejo. Com base nas informações originais, foram simulados dois sistemas de produção: ciclo de cria (Ccr) - mesmo manejo de descarte do rebanho 1; ciclo completo (Cco) - não ocorre venda à desmama e todos os machos, exceto os de reposição, são destinados ao abate.

Os preços médios dos componentes de produção (Tabela 2) para o rebanho 1 foram obtidos na propriedade e, para o rebanho 2, foram os descritos no Anualpec (2003).

Os componentes de custos anuais considerados para os dois rebanhos foram alimentação, formação e manutenção de pastagens, custos veterinários e outros custos (Tabela 2), de acordo com a categoria animal. Para o rebanho 1 , foram também incluídos os custos com suplementação na época seca ( silagem, concentrado), conforme descrito por Jorge Jr. et al. (2006). Foram computados também os custos adicionais relacionados à avaliação genética e outros custos relacionados ao melhoramento genético do rebanho. Esses últimos custos referem-se ao número adicional de funcionários com melhor remuneração, necessários para registro e digitação de informações de desempenho e avaliações visuais dos animais, à avaliação genética e emissão do Certificado Especial de Identificação e Produção - CEIP (empresa terceirizada).

Os componentes anuais de receita foram a venda de bezerros (as) à desmama, de fêmeas de descarte e de bois 
Tabela 1 - Parâmetros biológicos referentes aos rebanhos 1 e 2

Table 1 - Biological parameters used to describe herds 1 and 2

\begin{tabular}{|c|c|c|}
\hline $\begin{array}{l}\text { Parâmetro biológico } \\
\text { Biological parameter }\end{array}$ & $\begin{array}{c}\text { Rebanho } 1 \\
\text { Herd } 1\end{array}$ & $\begin{array}{c}\text { Rebanho } 2 \\
\text { Herd } 2\end{array}$ \\
\hline Número de matrizes (Number of breeding cows) & 10.500 & 10.500 \\
\hline Taxa de prenhez - novilhas (\%) (Pregnancy rate - heifers) & 85 & 75 \\
\hline Taxa de prenhez - vacas (\%)(Pregnancy rate - cows) & 80 & 70 \\
\hline Taxa de reposição (\%) (Replacement rate) & 25 & 37 \\
\hline Idade ao primeiro parto (meses) (Age at first calving, months) & 21 a 25 & 36 \\
\hline Número de doses por vaca (IA) (Number of doses per cow, AI) & 1,55 & 2 \\
\hline Mortalidade até a desmama (\%) (Mortality up to weaning, \%) & 4 & 8 \\
\hline Idade média à desmama (meses) (Average age at weaning, months) & 7 & 8 \\
\hline Peso médio da vaca adulta $(\mathrm{kg})$ (Average weight of mature cows) & 450 & 430 \\
\hline Peso médio do bezerro à desmama $(\mathrm{kg})$ (Average weaning weight - male calves) & 180 & 170 \\
\hline Peso médio da bezerra à desmama $(\mathrm{kg})$ (Average weaning weight - female calves) & 160 & 150 \\
\hline Peso médio ao abate dos machos $(\mathrm{kg})$ (Average slaughter weight) & 490 & 440 \\
\hline Idade ao abate (meses) (Slaughter age, months) & 24 & 36 \\
\hline Rendimento de carcaça (machos) (\%)(Dressing percentage, males) & 55 & 55 \\
\hline Rendimento de carcaça (vacas) (\%) (Dressing percentage, cows) & 48 & 48 \\
\hline
\end{tabular}

Tabela 2 - Preços médios dos componentes de produção para os rebanhos 1 e 2

Table 2 - Average prices of the production components (inputs and outputs)

\begin{tabular}{|c|c|c|}
\hline & $\begin{array}{c}\text { Rebanho } 1 \\
\text { Herd } 1\end{array}$ & $\begin{array}{c}\text { Rebanho } 2 \\
\text { Herd } 2\end{array}$ \\
\hline Componente de receita & Valores $(\mathrm{R} \$)$ & Valores $(\mathrm{R} \$)$ \\
\hline Outputs (Revenues) & Values & Values \\
\hline @ bois (@steers) _ & 56,00 & 56,00 \\
\hline Bezerros ( $\mathrm{R} \$ / \mathrm{kg}$ peso vivo) (Male calves, $R \$ / \mathrm{kg}$ live weight) & 3,00 & $2,23 * *$ \\
\hline Bezerras ( $\mathrm{kg} /$ peso vivo) (Female calves, $R \$ / \mathrm{kg}$ live weight) & 2,70 & $1,81 * *$ \\
\hline Novilhas sobreano ( $\mathrm{kg}$ de peso vivo)(Yearling heifers, $k g$ live weight) & 2,40 & $1,79 * *$ \\
\hline Novilhas vazias/descarte ( $\mathrm{kg}$ de peso vivo) (Culled not pregnant heifers, $\mathrm{kg}$ live weight) & 1,90 & $1,62 * *$ \\
\hline @Vacas descartadas (abate)@(Culled cows,slaughter) & 45,40 & $45,40 * *$ \\
\hline
\end{tabular}

Componente de custo

Inputs (Costs)

Alimentação (Feeding)

Pastagem - formação e manutenção (ha/ano) (Pastures - formation and maintenance, ha/year)

Sal mineral $(\mathrm{kg})$ (Mineral salt)

Custos veterinários (Veterinary costs)

Bezerras até 7 meses (R\$) (Female calves up to 7 months)

Novilhas (8 a 18 meses) (R\$) (Heifers, 8 to 18 months)

Novilhas (19 a 23 meses) (R\$) (Heifers, 19 to 23 months)

Vacas (R\$) (Cows)

Bezerros até 7 meses ( $\mathrm{R} \$$ ) (Calves up to 7 months)

Machos (8 a 21 meses) (R\$) (Males, 8 to 21 months)

Machos (21 a 48 meses) ( $\$$ ) (Males, 21 to 48 months)

$\begin{array}{rc}124,50 & 296,00^{* *} \\ 0,38 & 0,38 \\ & \\ 3,18 & 3,73^{* *} \\ 1,86 & 1,86 \\ 0,84 & 0,84 \\ 2,34 & 2,34 \\ 3,18 & 3,18 \\ 1,86 & - \\ - & 7,44 \\ & \\ 2,27 & - \\ 2,27 & - \\ 56,00 & - \\ 10,00 & 25,00 \\ 3,50 & 5,00 \\ 0,65 & 4,50\end{array}$

Custos com avaliação genética e outros custos (Genetic evaluation and other costs)

Avaliação de desmama ( $\mathrm{R} \$)$ (Weaning evaluation)

Avaliação de sobreano ( $\mathrm{R} \$$ ) (Yearling evaluation)

CEIP (certificado especial de identificação e produção) (R\$)(IPSC, identification and production special certificate)

Exame andrológico $(\mathrm{R} \$)^{*}$ (Andrologic exam)

Rastreabilidade (R\$) (Traceability)

Dose sêmen (R\$) (Dose semen)

${ }^{*}$ Componentes de produção exclusivos do sistema de CcoR (Production components exclusively for CbcR. system).

Dólar oficial médio em 2002: $1 \cup \$=R \$ 2,91$ (Annual average dollar currency - 2002: $1 \cup \$=R \$ 2.91$ ).

${ }^{* *}$ Anualpec (2003).

para abate. O rebanho 1 teve também como componente de receita a venda de novilhas ao sobreano, de sêmen e de tourinhos.
Foram escolhidas algumas características biológicas (Tabela 3) que poderiam influenciar os custos e as receitas dos dois sistemas de produção: 
rebanho 1 - para o ciclo de cria (Ccr), as características consideradas foram: peso à desmama $(\mathrm{PD})=$ peso médio do bezerro no ato da desmama, em torno dos 7 meses de idade; taxa de desmama (TD) = número de animais desmamados em relação ao número de vacas expostas à reprodução; e peso da vaca adulta $($ PVA $)=$ peso médio das fêmeas após seu primeiro parto. Para o ciclo completo (Cco) e o ciclo completo com venda de reprodutores (CcoR), além das características anteriores, foram considerados: o ganho médio diário no confinamento $(\mathrm{GMD})=$ ganho de peso no confinamento ( $\mathrm{kg})$ dividido pelo período do confinamento (90 dias); o peso ao abate $(\mathrm{PA})=$ peso médio dos machos castrados abatidos aos 24 meses após o confinamento e aos 36 meses, em regime de pastagem; o peso final dos tourinhos $(\mathrm{PFT})=$ peso médio dos machos não-castrados vendidos em leilão aos 24 meses após o período do confinamento; o peso da carcaça $(\mathrm{PC})=$ peso médio dos animais destinados ao abate multiplicado pelo rendimento de carcaça; o rendimento de carcaça (RC); e o consumo alimentar no confinamento (CA) = quantidade média da mistura silagem e concentrado (MS) ingerida no período de um dia. rebanho 2: para o ciclo completo com venda de reprodutores $(\mathrm{Ccr})$, as características consideradas foram: peso à desmama (PD); taxa de desmama (TD); e peso da vaca adulta (PVA). Para o ciclo completo (Cco), além destas características, foram considerados: o peso ao abate (PA); o peso da carcaça (PC); o rendimento de carcaça (RC); e o consumo alimentar no confinamento (CA) para os machos.

Para o cálculo dos valores econômicos, utilizou-se o modelo bioeconômico desenvolvido por Jorge Jr. et al. (2006). Foram simulados os diferentes sistemas de produção para obtenção de seus desempenhos, seus custos e suas receitas. Assim, o aumento do desempenho dos animais atribuído à seleção não acarretará redução no tamanho do rebanhoe, portanto, os custos fixos permanecem inalterados e não precisam ser considerados no cálculo dos valores econômicos.

Para avaliação do impacto das mudanças no desempenho das características sobre o lucro anual dos sistemas de produção, os valores iniciais de cada característica foram aumentados em 1\%, mantendo-se as outras características constantes. Considerando dados descritos por Groen et al.

Tabela 3 - Características biológicas que afetam as receitas e os custos dos sistemas de produção Table 3 - Biological traits affecting the revenues and costs of production systems

\begin{tabular}{lcc}
\hline Produto & Classe animal & Característica do objetivo de seleção \\
Product & Animal class & Traits in the breeding objectives
\end{tabular}

Receita

Revenue

\begin{tabular}{|c|c|c|}
\hline Bezerros à desmama & Bezerro (a) & Peso do bezerro à desmama (PD) \\
\hline Male and female calves at weaning & Male and female calves & $\begin{array}{l}\text { Weaning weight (male and female calves) (WC) } \\
\text { Taxa de desmama (TD) } \\
\text { Weaning rate }(W R)\end{array}$ \\
\hline
\end{tabular}

Vacas de descarte $\quad$ Vacas $\quad$ Peso à idade adulta (PVA)

Culled cows Cows Mature weight-cows (MW)

Bois para abate $\quad$ Bois $\quad$ Ganho médio diário no confinamento $\left(\mathrm{GMD}_{\mathrm{C}}\right)^{*} \mathrm{a}$

Steers (slaughter) Steers Average daily gain infeed-lot $*\left(D_{A} G_{F L}\right)$

Peso abate (PA)

Slaughter weight(SW)

Rendimento carcaça (RC)

Dressing percentage $(Y G)$

$\begin{array}{lll}\text { Tourinhos } & \text { Tourinhos } & \text { Peso ao final dos tourinhos* (PFT) } \\ \text { Young bulls } & \text { Young bulls } & \text { Final weight- young bulls*(FWYB) }\end{array}$

Custos

Costs

\begin{tabular}{lcl}
\hline $\begin{array}{l}\text { Alimentação } \\
\text { Feeding }\end{array}$ & $\begin{array}{c}\text { Bois } \\
\text { Steers }\end{array}$ & $\begin{array}{l}\text { Consumo no confinamento* }\left(\mathrm{C}_{\mathrm{conf}}\right) \\
\text { Feed intake infeed-lot* }\left(F I_{F I}\right) \\
\text { Consumo na pastagem** }\left(\mathrm{C}_{\mathrm{seca}}\right) \\
\text { Feed intake at pasture } * *\left(F I_{P}\right)\end{array}$ \\
$\begin{array}{ll}\text { Veterinários } \\
\text { Veterinary costs }\end{array}$ & Bezerros, novilhas, vacas, bois, tourinhos & Taxa de desmama (TD) \\
\hline
\end{tabular}

* Para o rebanho 1 (For herd 1).

** Para o rebanho 2 (For herd 2). 
(1997), os valores econômicos (VE) foram calculados como a diferença entre o lucro após a seleção e o lucro antes da seleção, o qual resultou no lucro marginal (LM), a partir de:

$$
\mathrm{VE}=(1 / \mathrm{n}) \times(\mathrm{LM} / \mathrm{DD})
$$

em que DD é a diferença marginal no desempenho da característica (lucro após a seleção - lucro antes da seleção) e n, o número de animais referentes a cada categoria.

Para avaliar o impacto de mudanças nos preços dos insumos e produtos sobre os valores econômicos das características, foram realizadas análises de sensibilidade, com variação nos preços de venda dos bezerros, da arroba da vaca e do boi gordo, do custo com pastagens e do custo do concentrado e da silagem em $\pm 20 \%$ e $\pm 40 \%$.

\section{Resultados e Discussão}

Como demonstrado na Tabela 4, com exceção do consumo alimentar (confinamento e pastagem) e do peso da vaca adulta no ciclo completo (rebanho 2), todas as características apresentaram valores econômicos positivos nos dois sistemas de produção. Esse resultado era esperado, pois o aumento no consumo alimentar eleva os custos com alimentação. Bittencourt (2001) obteve custo com alimentação de-US $\$ 4,40 /$ vaca/ano em um rebanho de ciclo completo com manejo exclusivamente a pasto.

Os valores econômicos do peso à desmama foram iguais nos dois sistemas de produção no $\mathrm{R} 1(\mathrm{R} \$ 0,40)$ e superiores ao obtido para o $\mathrm{R} 2(\mathrm{R} \$ 0,34)$. Os maiores valores

Tabela 4 - Valores econômicos (VE) expressos por unidade da característica para os ciclos completo (CCo) e completo com venda de reprodutores ( $\mathrm{CcoR}$ )

Table 4 - Economic values (EV) of traits for complete breeding cycle $(\mathrm{Cbc})$ and complete breeding cycle with sales of animals for reproduction $(\mathrm{CbcR})$

\begin{tabular}{|c|c|c|c|}
\hline & $\begin{array}{l}\operatorname{Cco}\left(\mathrm{R}_{1}\right) \\
C b c(H 1)\end{array}$ & $\begin{array}{l}\mathrm{Cco}\left(\mathrm{R}_{2}\right) \\
\mathrm{Cbc}\left(\mathrm{H}_{2}\right)\end{array}$ & $\begin{array}{c}\operatorname{CcoR}\left(\mathrm{R}_{1}\right) \\
C b c R(H 1)\end{array}$ \\
\hline $\mathrm{PD}(\mathrm{kg})(W W, k g)$ & 0,40 & 0,34 & 0,40 \\
\hline $\mathrm{TD}(\%)(W R, \%)$ & 4,20 & 3,51 & 10,15 \\
\hline $\operatorname{PVA}(\mathrm{kg})(M W, k g)$ & 0,09 & $-0,16$ & 0,09 \\
\hline $\operatorname{GMDc}(\mathrm{g})\left(D A G_{F L}\right)$ & 0,32 & - & 0,76 \\
\hline $\mathrm{PA}(\mathrm{kg})(S W, k g)$ & 1,17 & 1,09 & 1,17 \\
\hline $\operatorname{PFT}(\mathrm{kg})(F W Y B, k g)$ & - & - & 11,85 \\
\hline $\mathrm{PC}(\mathrm{kg})(C W, \mathrm{~kg})$ & 2,19 & 2,03 & 2,19 \\
\hline $\mathrm{RC}(\%)(Y G, \%)$ & 28,61 & 23,89 & 28,61 \\
\hline CAc (kg MS/dia) $\left(F I_{F L}, k g D M / d a y\right)$ & $-0,45$ & - & $-0,45$ \\
\hline CAp (kg MS/dia) $\left(F I_{P}, k g D M /\right.$ day $)$ & - & $-0,03$ & - \\
\hline
\end{tabular}

PD - peso à desmama; TD - taxa de desmama; PAV - peso da vaca adulta; GMD - ganho médio diário no confinamento; PA - peso ao abate; PFT - peso final dos tourinhos; PC - peso da carcaça; RC - rendimento da carcaça; CA - consumo alimentar no confinamento; Cap - consumo alimentar na pastagem.

$W W$ - weaning weight; WR - weaning rate; $M W$ - mature weight; $D A G_{F L}$-average daily gain in feedlot; $S W$-slaughter weight; $F W Y B$ - final weight - young bulls; $C W$ - carcass weight; $D P$ - dressing percentage; $F I_{F L}$ - feed intake( feedlot); $F I_{P}$ - feed intake (pasture). econômicos obtidos no rebanho 1 são reflexo de seu menor custo com pastagem e dos maiores preços de venda dos animais comercializados nesta fase. Brumatti (2002), trabalhado com animais zebus no Brasil, relatou valores de R\$ 0,63 para esta característica.

O aumento do peso da vaca à idade adulta no rebanho 1 afetou de forma discreta o lucro e resultou em valores econômicos positivos mas próximos de zero ( $\mathrm{R}$ \$ 0,09), o que pode ser explicado pelo baixo custo com manutenção de pastagens. Assim, a princípio, a seleção para aumento de peso em fêmeas adultas não apresentaria efeito negativo no lucro da fazenda. Todavia, este resultado deve ser analisado com cuidado, uma vez que, neste estudo, supôs-se haver disponibilidade de áreas adicionais para comportar fêmeas mais pesadas em pastagens da própria fazenda, o que pode não ser realístico em longo prazo. Para o rebanho 2, o aumento em $1 \mathrm{~kg}$ do PVA resultou em valor econômico negativo (-R \$ 0,16), o que poderia acarretar prejuízos neste tipo de sistema de produção. Contudo, estudos adicionais envolvendo a resposta esperada à seleção devem ser realizados para avaliação do impacto da inclusão dessa característica em índices de seleção.

O maior valor econômico para taxa de desmama foi obtido no ciclo completo com venda de reprodutores ( $\mathrm{R} \$ 10,15)$, comprovando o impacto desta característica no número de tourinhos vendidos em leilão, os quais possuíam um valor agregado bem superior ao dos animais vendidos para abate.

Os valores econômicos do peso ao abate foram iguais no rebanho $1(\mathrm{R} \$ 1,17)$ para os dois ciclos e superiores aos do rebanho 2 ( $\mathrm{R} \$ 1,09)$. Os custos mais altos com o confinamento no rebanho 1 foram compensados pelo menor tempo de permanência dos machos no rebanho (24 meses) em comparação ao rebanho 2 (36 meses) e pelos seus menores custos com formação e manutenção de pastagens. O valor econômico do peso final dos tourinhos (PFT), calculado somente para o sistema CcoR, foi de $\mathrm{R} \$ 11,85$.Essa característica poderia ser considerada equivalente ao peso ao abate, uma vez que se refere ao peso atingido no final do confinamento, quando os outros machos são enviados ao abate. Embora teoricamente os valores econômicos sejam calculados para as circunstâncias de produção de rebanhos comerciais, no Brasil, alguns rebanhos com número muito grande de animais possuem seus próprios programas de seleção com venda de reprodutores e, ao mesmo tempo, destinam uma parte significativa de seus animais para produção de carne.Esse valor econômico foi calculado para demonstrar a importância econômica desta característica em rebanhos nos quais é desenvolvido um programa de seleção, o qual 
acarreta custos adicionais com obtenção de dados e a avaliação genética, mas resulta em maior valor de venda pela certificação dos animais por meio de suas DEP. Neste estudo, a receita anual do ciclo completo com venda de reprodutores, CcoR (rebanho 1), foi de $\mathrm{R} \$ 8.773 .768,00$, enquanto o custo anual total foi de $\mathrm{R} \$ 2.183 .150,00$. Desse total, os custos com melhoramento genético na propriedade foram de $\mathrm{R} \$ 109.321,00$.

O valor econômico do ganho médio diário, calculado somente para o rebanho 1 , foi maior para o ciclo completo com venda de reprodutores $(\mathrm{R} \$ 0,76)$ que para o ciclo completo ( $\mathrm{R} \$ 0,32)$. Esses resultados também são reflexo das suposições do modelo, no qual animais com ganhos maiores no confinamento apresentam maiores pesos ao abate (Cco) ou maiores pesos finais dos tourinhos a serem vendidos em leilão.

O rendimento de carcaça foi a característica que apresentou maiores valores econômicos no ciclo completo, de $\mathrm{R} \$ 28,61$ para rebanho 1 e de $\mathrm{R} \$ 23,89$ para rebanho 2.Esses elevados valores econômicos podem ser explicados pela importância da característica para os dois sistemas, nos quais não houve um aumento de custos associado ao aumento nos níveis iniciais desta característica. Brumatti (2002) obteve valor econômico de R\$ 14,28 para esta característica. Quando o valor econômico foi calculado para o peso da carcaça, foram obtidos valores de $\mathrm{R} \$ 2,19$ (rebanho 1) e R \$2,03 (rebanho 2).Bittencourt (2001) obteve valor econômico de US\$1,00 para a mesma característica. Por não apresentar custos associados ao seu aumento de desempenho, a característica rendimento de carcaça representa grande importância econômica em rebanhos de ciclo completo. Todavia, estudos adicionais devem ser realizados para avaliação da viabilidade de inclusão desta característica em índices de seleção. Talvez fosse mais realístico utilizar o peso da carcaça, que é determinado pelo peso ao abate e pelo rendimento da carcaça.

Com exceção do peso da vaca à idade adulta (rebanho 2), todas as características relacionadas ao ciclo de cria apresentaram valores econômicos positivos (Tabela 5). O peso à desmama teve maior impacto no lucro no ciclo de cria (Ccr) no rebanho 1 ( $\mathrm{R} \$ 1,31)$, em decorrência de seus menores custos com alimentação e dos maiores valores de comercialização dos bezerros em comparação ao rebanho 2 $(\mathrm{R} \$ 1,16)$. Os valores econômicos do peso à desmama para o ciclo de cria foram maiores que os obtidos no ciclo completo e no ciclo completo com venda de reprodutores, comprovando a maior importância desta característica neste sistema de produção. Bittencourt (2001), trabalhando com um rebanho de cria, obteve valor econômico de US\$ 0,29/ vaca/ano para o peso à desmama.
Tabela 5 - Valores econômicos expressos por unidade da característica para o ciclo de cria (Ccr) nos rebanhos 1 e 2

Table 5 - Economic values of traits for cow-calf cycle $\left(C C_{c}\right)$ in herds 1 and 2

\begin{tabular}{|c|c|c|}
\hline & \multicolumn{2}{|c|}{$\begin{array}{c}\text { Valor econômico }(\mathrm{R} \$) \\
\text { Economic value }(R \$)\end{array}$} \\
\hline & $\begin{array}{l}\mathrm{Ccr}(\mathrm{R} 1) \\
\mathrm{CC}_{C}(\mathrm{H} 1) \\
\end{array}$ & $\begin{array}{l}\mathrm{Ccr}(\mathrm{R} 2) \\
\mathrm{CC}_{C}(\mathrm{H} 2) \\
\end{array}$ \\
\hline Peso à desmama (kg) & 1,31 & 1,16 \\
\hline $\begin{array}{l}\text { Weaning weight }(\mathrm{kg}) \\
\text { Taxa de desmama }(\%) \\
\text { Weaning rate }(\%)\end{array}$ & 3,36 & 2,74 \\
\hline $\begin{array}{l}\text { Peso da vaca adulta }(\mathrm{kg}) \\
\text { Mature weight }(\mathrm{kg})\end{array}$ & 0,09 & $-0,16$ \\
\hline
\end{tabular}

O aumento da taxa de desmama apresentou maior efeito no lucro no rebanho 1. Comparando os valores do ciclo completo $(\mathrm{R} \$ 4,20$ para o rebanho 1 e $\mathrm{R} \$ 3,51$ para o rebanho 2) e do ciclo completo com venda de reprodutores ( R \$10,15), observa-se que os valores econômicos calculados para o ciclo de cria foram menores, pois os animais do Cco e CcoR possuem valor agregado superior ao daqueles vendidos à desmama.

Os valores econômicos para o peso da vaca à idade adulta para o ciclo de cria apresentaram o mesmo efeito que no ciclo completo nos rebanhos 1 e 2 , com valores positivos no rebanho 1 e negativos para o rebanho $2(\mathrm{R} \$ 0,09 ;-\mathrm{R} \$ 0,16$, respectivamente).

Valores econômicos são específicos para cada contexto de produção e de mercado. Assim, não é possível comparar resultados de valores econômicos de uma mesma característica obtidos em regiões ou países diferentes.

Para analisar o comportamento dos valores econômicos sob diferentes situações de preços de componentes de produção, procedeu-se à variação dos preços do bezerro, das arrobas do boi e da vaca, do concentrado e dos custos com produção de silagem e formação/manutenção de pastagens em $\pm 20 \%$ e $\pm 40 \%$.

Quando o preço da arroba da vaca foi modificado, as variações no valor econômico do peso da vaca à idade adulta foram de $44 \%$ no rebanho 1 e de $50 \%$ no rebanho 2 . Essa variação maior (50\% no rebanho 2) reflete o custo mais elevado com manutenção dessas fêmeas em pastagem neste rebanho (Tabelas 6 e 7).

A mudança no preço da pastagem ocasionou maiores diferenças nos valores econômicos no rebanho 2. No caso do peso à desmama, os resultados apresentaram variações de $20 \%$ no rebanho 1 e de $28 \%$ no rebanho 2 . Para taxa de desmama, as variações foram de $10 \%$ no rebanho 1 e de $20 \%$ no rebanho 2 e a mesma diferença em porcentagem ocorreu 
Tabela 6 - Análise de sensibilidade para avaliar o efeito de mudanças no preço do kg bezerro (ou no preço da @ vaca) e no custo de pastagens sobre os valores econômicos das características peso à desmama (PD), taxa de desmama (TD) e peso da vaca adulta (PVA) para o ciclo de cria nos rebanhos 1 e 2

Table 6 - Sensitivity analysis for the effect of changes in prices of calves (or price @ cow) and cost of pastures on economic values of weaning weight $(W W)$, weaning rate (WR) and mature weight (MW) for cow-calf cycle in herds 1 and 2

\begin{tabular}{|c|c|c|c|c|c|c|}
\hline & \multicolumn{2}{|c|}{$\begin{array}{c}\mathrm{PD}(\mathrm{R} \$ / \mathrm{kg} / \text { bezerro }) \\
W W(R \$ / \mathrm{kg} / \text { calf })\end{array}$} & \multicolumn{2}{|c|}{$\begin{array}{c}\mathrm{TD}(\mathrm{R} \$ / 1 \% / \mathrm{vaca}) \\
W R(R \$ / 1 \% / \text { cow })\end{array}$} & \multicolumn{2}{|c|}{$\begin{array}{c}\mathrm{PVA}(\mathrm{RS} / \mathrm{kg} / \mathrm{vaca}) \\
M W(R \$ / \mathrm{kg} / \text { cow })\end{array}$} \\
\hline & $\begin{array}{l}\mathrm{R} 1 \\
\mathrm{H} 1\end{array}$ & $\begin{array}{l}\mathrm{R} 2 \\
\mathrm{H} 2\end{array}$ & $\begin{array}{l}\mathrm{R} 1 \\
\mathrm{H} 1\end{array}$ & $\begin{array}{l}\mathrm{R} 2 \\
\mathrm{H} 2\end{array}$ & $\begin{array}{l}\mathrm{R} 1 \\
\mathrm{H} 1\end{array}$ & $\begin{array}{l}\mathrm{R} 2 \\
\mathrm{H} 2\end{array}$ \\
\hline $\begin{array}{l}\text { Base } \\
\text { Base }\end{array}$ & 1,31 & 1,16 & 3,36 & 2,74 & 0,09 & $-0,16$ \\
\hline \multicolumn{7}{|c|}{ Preço do bezerroa (PD e TD) e @ vacab (PAV) } \\
\hline $\begin{array}{l}-40 \% \\
-20 \% \\
+20 \% \\
+40 \% \\
\end{array}$ & $\begin{array}{l}1,01 \\
1,16 \\
1,46 \\
1,63 \\
\end{array}$ & $\begin{array}{l}0,88 \\
1,02 \\
1,29 \\
1,42 \\
\end{array}$ & $\begin{array}{l}1,76 \\
2,56 \\
4,16 \\
4,96 \\
\end{array}$ & $\begin{array}{l}1,65 \\
2,17 \\
3,28 \\
3,83 \\
\end{array}$ & $\begin{array}{l}0,05 \\
0,07 \\
0,11 \\
0,13 \\
\end{array}$ & $\begin{array}{l}-0,08 \\
-0,12 \\
-0,20 \\
-0,24 \\
\end{array}$ \\
\hline \multicolumn{7}{|c|}{ Custo da pastagem } \\
\hline $\begin{array}{l}-40 \% \\
-20 \% \\
+20 \% \\
+40 \%\end{array}$ & $\begin{array}{l}1,05 \\
1,18 \\
1,45 \\
1,58\end{array}$ & $\begin{array}{l}0,84 \\
1,00 \\
1,33 \\
1,49\end{array}$ & $\begin{array}{l}3,69 \\
3,53 \\
3,20 \\
3,08\end{array}$ & $\begin{array}{l}3,30 \\
3,02 \\
2,45 \\
2,17\end{array}$ & $\begin{array}{l}0,11 \\
0,10 \\
0,08 \\
0,07\end{array}$ & $\begin{array}{l}-0,10 \\
-0,13 \\
-0,18 \\
-0,21\end{array}$ \\
\hline
\end{tabular}

para o PVA (22 a 33\%) para os rebanhos 1 e 2 respectivamente. Essas diferenças de valores podem ser atribuídas ao consumo alimentar no rebanho 2, pois, neste sistema de produção, os animais são mantidos em regime de pastejo durante toda sua permanência no rebanho, acarretando custos mais elevados com alimentação.

As variações no preço da arroba do boi influenciaram de forma mais significativa o valor econômico do peso ao abate no rebanho 1 (Tabela 7), o que pode ser explicado pelo maior valor da arroba do boi neste rebanho.

Maiores variações no valor econômico do peso ao abate foram observadas no rebanho 2 (em torno de $36 \%$ ) em comparação ao rebanho 1 (24\%), em decorrência do maior custo de pastagem neste rebanho, influenciando de uma forma mais significativa o valor econômico do peso ao abate. O efeito do maior custo de pastagem foi mais acentuado no valor econômico do consumo alimentar em pastagem (CAp), que apresentou variações em torno de $50 \%$. As mudanças nos preços da arroba do boi afetaram os valores econômicos do consumo alimentar em pastagem, que apresentou variações de $29 \%$ no rebanho 1 e de $37 \%$ no rebanho 2. Novamente, essas variações podem ser explicadas pela importância do consumo alimentar neste sistema de produção, no qual os animais são mantidos a pasto, com custos mais elevados durante toda sua permanência no rebanho.

O efeito das mudanças nos preços do concentrado e da silagem (rebanho 1) sobre os valores econômicos do peso ao abate foi semelhante em ambos os casos e ocasionou variações nos valores de 17 e $20 \%$, respectivamente. Para o valores econômicos do consumo alimentar no confinamento (CAC), essas variações foram de 40 e $48 \%$, respectivamente. De modo geral, as mudanças dos preços dos produtos e insumos tiveram impacto maior sobre os valores econômicos das características nos sistemas de produção nos quais esses preços, na situação básica, eram mais elevados (rebanho 1).

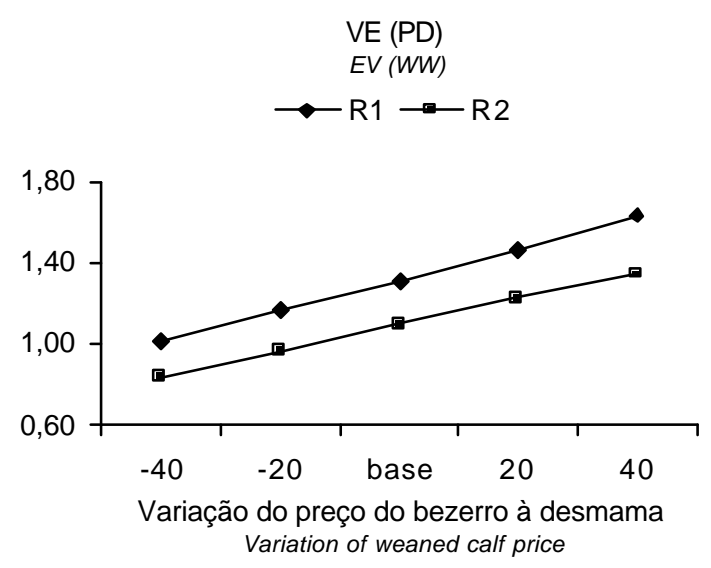

Figura 1 - Efeito da variação do preço do bezerro à desmama sobre o valor econômico (VE) do peso à desmama (PD) no ciclo de cria, para os rebanhos 1 e 2 (R1 e R2).

Figure 1 - Effect of variation of weaned calf price on the economic value (EV) of weaning weight (WW) for the cow-calf cycle in herds 1 and 2 ( $H 1$ and $H 2)$. 
Tabela 7 - Análises de sensibilidade para avaliar os efeitos de mudanças no preço da @boi e nos custos de pastagem, concentrado e silagem sobre os valores econômicos das características peso ao abate (PA) e consumo alimentar (CAp e CAc) no ciclo completo, para os rebanhos 1 e 2

Table 7 - Sensitivity analyses for the effect of changes in prices@ steers and cost of pastures, concentrate and silage on economic values of WS and feed intake $\left(F I_{P}\right.$ and $\left.F I_{F L}\right)$ for complete breeding cycle in herds 1 and 2

\begin{tabular}{|c|c|c|c|c|}
\hline & \multicolumn{2}{|c|}{$\begin{array}{l}\mathrm{PA}(\mathrm{R} \$ / \mathrm{kg} / \mathrm{boi}) \\
W S(R \$ / \mathrm{kg} / \text { steers })\end{array}$} & \multicolumn{2}{|c|}{$\begin{array}{l}\text { Consumo* } \\
\text { Feed intake }\end{array}$} \\
\hline & $\begin{array}{l}\mathrm{R} 1 \\
\mathrm{H} 1\end{array}$ & $\begin{array}{l}\mathrm{R} 2 \\
\mathrm{H} 2\end{array}$ & $\begin{array}{l}\mathrm{R} 1 \\
\mathrm{H} 1\end{array}$ & $\begin{array}{l}\mathrm{R} 2 \\
H 2\end{array}$ \\
\hline $\begin{array}{l}\text { Base } \\
\text { Base }\end{array}$ & 1,17 & 1,09 & $-0,45$ & $-0,030$ \\
\hline
\end{tabular}

@boi (@steers)

\begin{tabular}{lllll}
$-40 \%$ & 0,61 & 0,64 & $-0,59$ & $-0,042$ \\
$-20 \%$ & 0,89 & 0,86 & $-0,52$ & $-0,039$ \\
$+20 \%$ & 1,45 & 1,31 & $-0,38$ & $-0,024$ \\
$+40 \%$ & 1,73 & 1,54 & $-0,31$ & $-0,018$ \\
\hline
\end{tabular}

Custo da pastagem (Cost of pasture)

\begin{tabular}{llllr}
$-40 \%$ & 1,45 & 1,48 & - & $-0,042$ \\
$-20 \%$ & 1,31 & 1,29 & - & $-0,039$ \\
$+20 \%$ & 1,03 & 0,91 & - & $-0,024$ \\
$+40 \%$ & 0,89 & 0,75 & - & $-0,018$ \\
\hline
\end{tabular}

Custo do concentrado (Cost of concentrate)

\begin{tabular}{lllll}
$-40 \%$ & 1,37 & - & $-0,23$ & - \\
$-20 \%$ & 1,27 & - & $-0,34$ & - \\
$+20 \%$ & 1,07 & - & $-0,56$ & - \\
$+40 \%$ & 0,97 & - & $-0,67$ & - \\
\hline
\end{tabular}

Custo silagem (Cost of silage)

\begin{tabular}{lllll}
$-40 \%$ & 1,41 & - & $-0,27$ & - \\
$-20 \%$ & 1,29 & - & $-0,36$ & - \\
$+20 \%$ & 1,05 & - & $-0,54$ & - \\
$+40 \%$ & 0,93 & - & $-0,63$ & - \\
\hline
\end{tabular}

* Para o rebanho 1, a característica é consumo de alimentos no confinamento (CAC), enquanto, para o rebanho 2 , a característica é consumo de pastagens (CAP)

* In herd 1, the trait is feed-intake at feed-lot $\left(F I_{F L}\right)$ and in herd 2 it is feed-intake at pasture $\left(F I_{P}\right)$

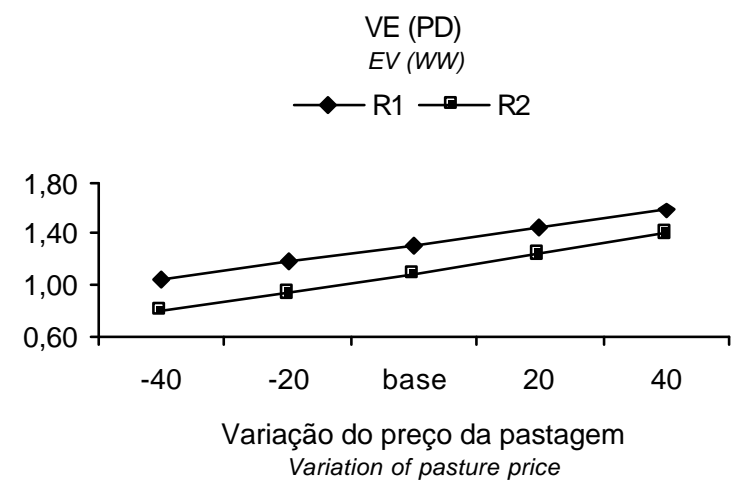

Figura 2 - Efeito da variação do preço da pastagem sobre o valor econômico (VE) do peso à desmama (PD) no ciclo de cria, para os rebanhos 1 e 2 (R1 e R2).

Figure 2 - Effect of variation of pasture price on the economic value (EV) of weaning weight (WW) for the cow-calf cycle in herds 1 and 2 ( $H 1$ and $H 2)$.

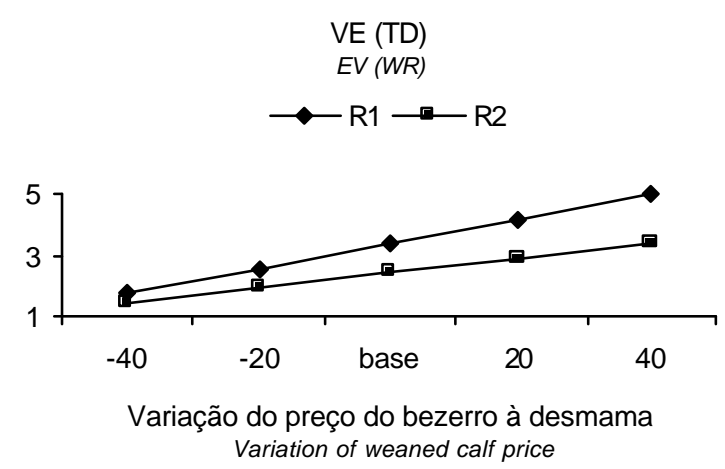

Figura 3 - Efeito da variação do preço do bezerro à desmama sobre o valor econômico (VE) da taxa de desmama (TD) no ciclo de cria, para os rebanhos 1 e 2 (R1 e R2).

Figure 3 - Effect of variation of weaned calf price on the economic value $(E V)$ of weaning rate (WR) for the cow-calf cycle in herds 1 and 2 ( $H 1$ and $H 2)$.

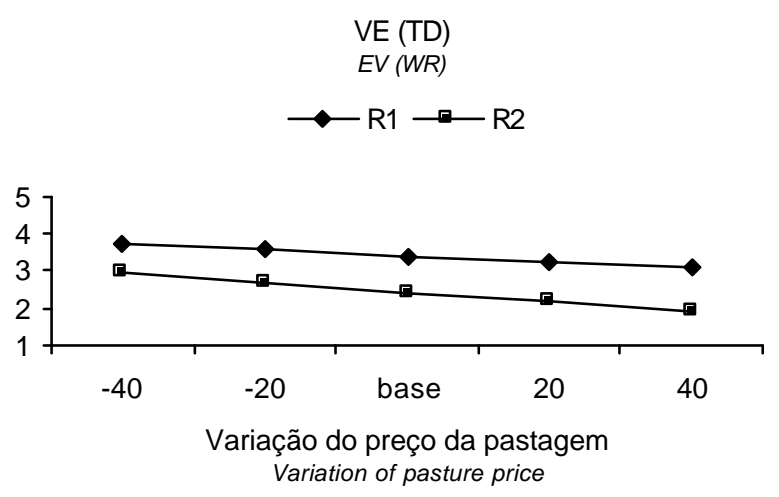

Figura 4 - Efeito da variação do preço da pastagem sobre o valor econômico (VE) da taxa de desmama (TD) no ciclo de cria, para os rebanhos 1 e 2 (R1 e R2).

Figure 4 - Effect of variation of pasture price on the economic value (EV) of weaning rate (WR) for the cow-calf cycle in herds 1 and 2 (H1 and H2).
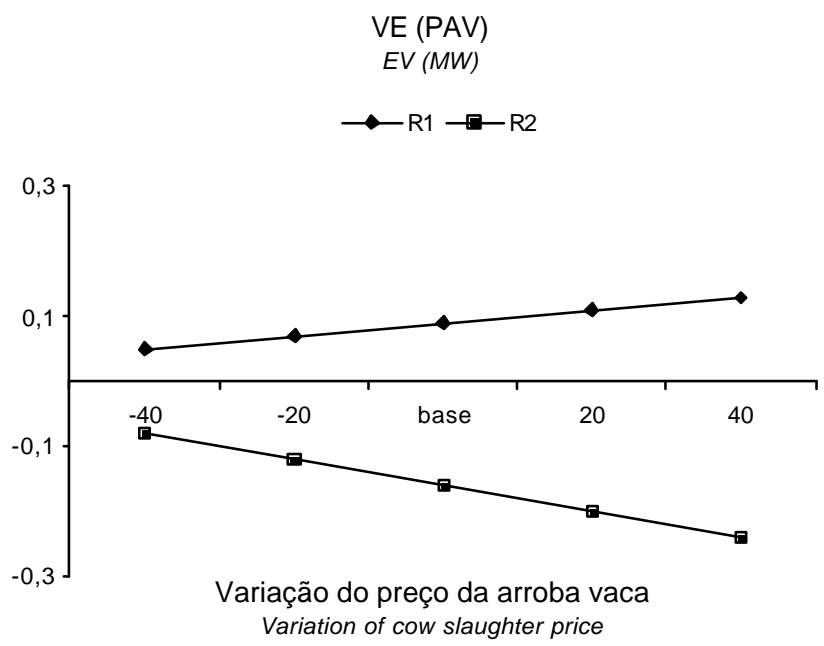

Figura 5 - Efeito da variação do preço da arroba da vaca sobre o valor econômico (VE) do peso adulto da vaca (PAV) no ciclo de cria, para os rebanhos 1 e 2 (R1 e R2).

Figure 5 - Effect of variation of cow slaughter price on the economic value (EV) of mature weight (MW) for the cow-calf cycle in herds 1 and 2 ( $\mathrm{H} 1$ and $\mathrm{H} 2$ ). 
Os efeitos das variações dos preços dos produtos e insumos sobre os valores econômicos não foram proporcionais, ou seja, variações de $\pm 20 \%$ ou $\pm 40 \%$ no preço dos produtos e insumos não acarretaram as mesmas variações nos valores econômicos das características (Figuras 1 a 11).

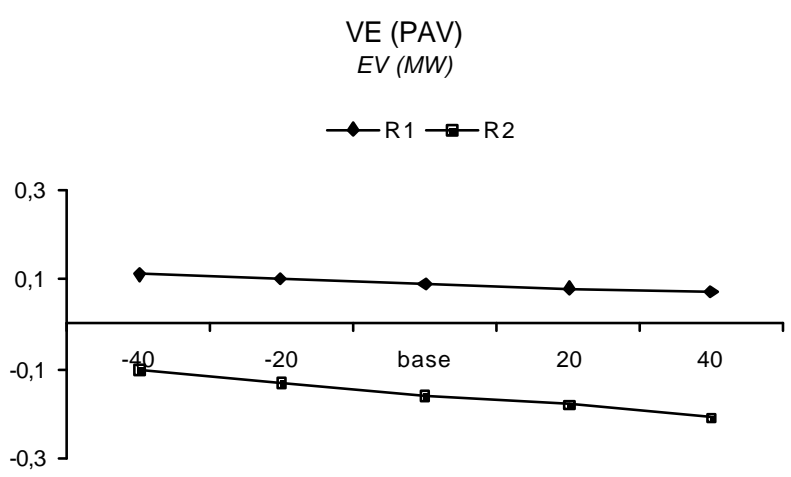

Variação do preço da pastagem Variation of pasture price

Figura 6 - Efeito da variação do preço da pastagem sobre o valor econômico (VE) do peso adulto da vaca (PAV) no ciclo de cria, para os rebanhos 1 e 2 (R1 e R2).

Figure 6 - Effect of variation of pasture price on the economic value (EV) of mature weight (MW) for the cow-calf cycle in herds 1 and 2 (H1 and H2).

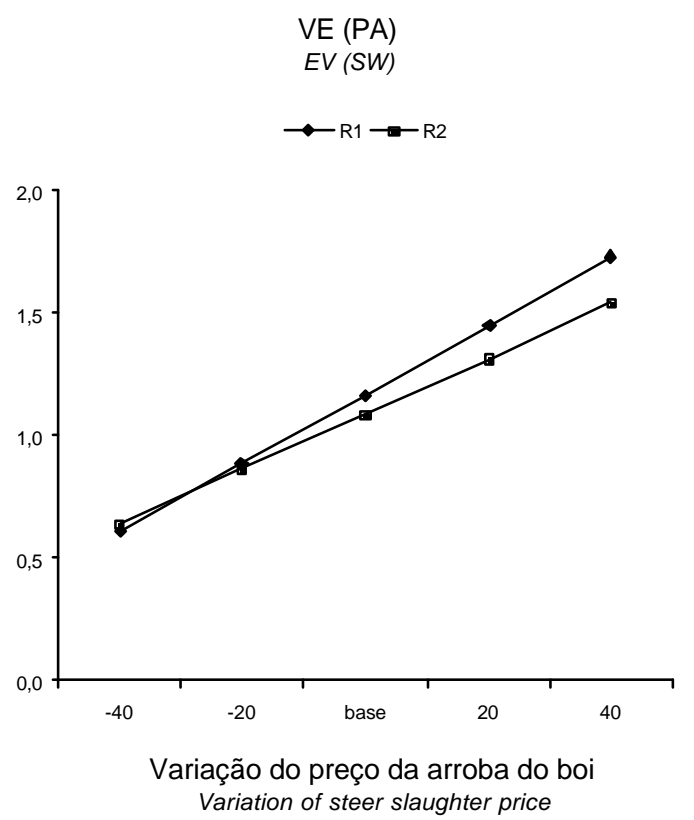

Figura 7 - Efeito da variação do preço da arroba do boi sobre o valor econômico (VE) do peso ao abate (PA) no ciclo completo, para os rebanhos 1 e 2 (R1 e R2).

Figure 7 - Effect of variation of steer slaughter price on the economic value (EV) of weight at slaughter (SW) for the complete breeding cycle in herds 1 and 2 ( $\mathrm{H} 1$ and $\mathrm{H} 2$ ).

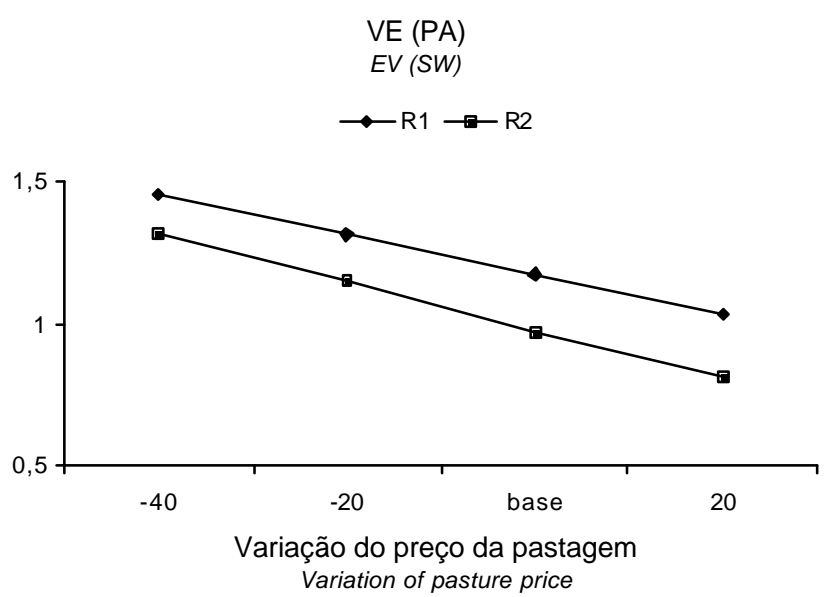

Figura 8 - Efeito da variação do preço da pastagem sobre o valor econômico (VE) do peso ao abate (PA) no ciclo completo, para os rebanhos 1 e 2 (R1 e R2).

Figure 8 - Effect of variation of pasture price on the economic value (EV) of weight at slaughter (SW), for the complete breeding cycle, in herds 1 and 2 ( $\mathrm{H} 1$ and $\mathrm{H} 2$ ).
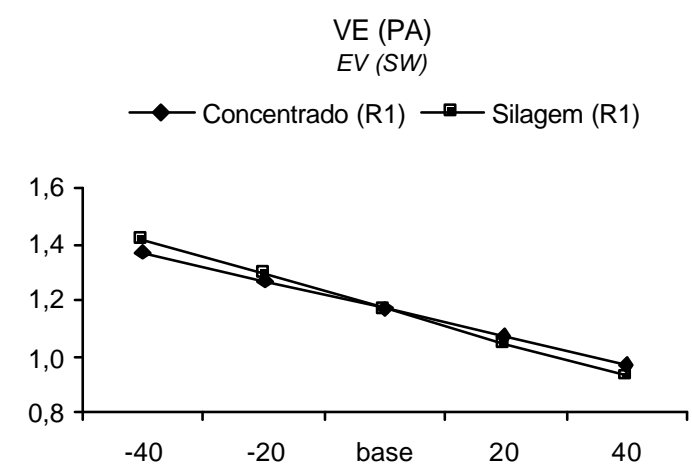

Variação dos preços da silagem e do concentrado Variation of concentrate and silage prices

Figura 9 - Efeito da variação do preço da silagem e do concentrado sobre o valor econômico (VE) do peso ao abate (PA) no ciclo completo para o rebanho 1 (R1).

Figure 9 - Effect of variation of concentrate and silage prices on the economic value $(E V)$ of weight at slaughter (SW) for the complete breeding cycle in herd $1(\mathrm{H} 1)$.

$$
\begin{gathered}
\mathrm{VE}(\mathrm{CA}) \\
E V(F I)
\end{gathered}
$$

$\longrightarrow$ Silagem + Concentrado (R1)

$\rightarrow$ Pastagem (R2)

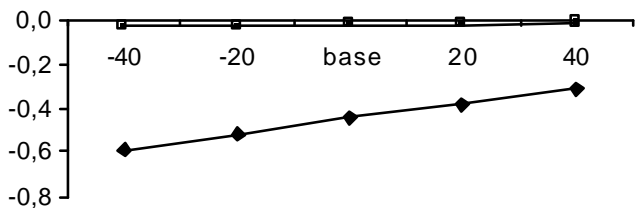

Variação do preço da arroba do boi Variation of steer slaughter price

Figura 10 - Efeito da variação do preço da arroba do boi sobre o valor econômico (VE) do consumo alimentar (CA), para os rebanhos 1 e 2 (R1 e R2).

Figure 10 - Effect of variation of steer slaughter price on the economic value (EV) of feed intake (FI), for the complete breeding cycle in herds 1 and 2 ( $\mathrm{H} 1$ and $\mathrm{H} 2$ ). 


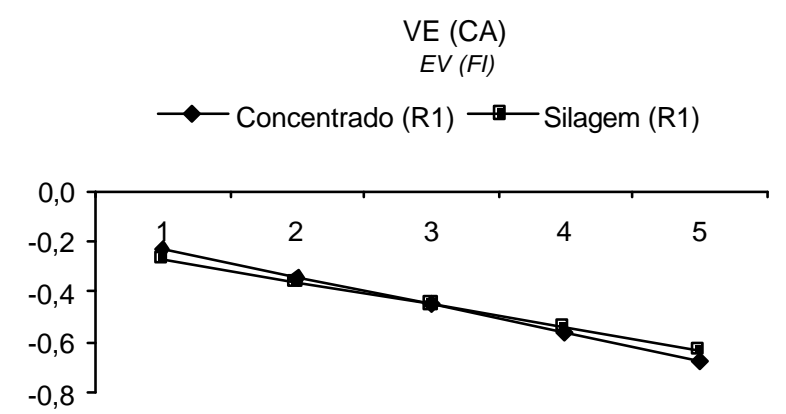

Variação dos preços da silagem e do concentrado Variation of concentrate and silage prices

Figura 11 - Efeito da variação do preço da silagem e do concentrado sobre o valor econômico (VE) do consumo alimentar (CA) no ciclo completo, para os rebanhos 1 e 2 (R1 e R2).

Figure 11 - Effect of variation of concentrate and silage prices on the economic value $(E V)$ of feed intake (FI), for the complete breeding cycle in herds 1 and 2 ( $\mathrm{H} 1$ and $\mathrm{H} 2$ ).

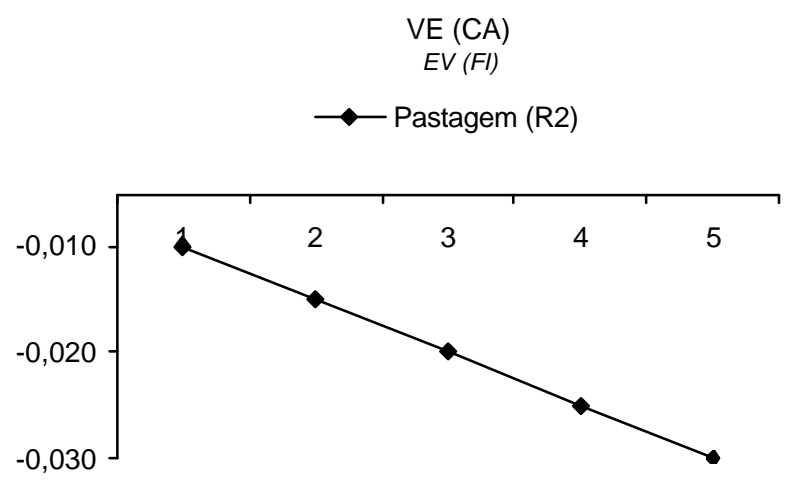

Variação do preço da pastagem Variation of pasture price

Figura 12 - Efeito da variação do preço da pastagem sobre o valor econômico (VE) do consumo alimentar (CA) no ciclo completo.

Figure 12 - Effect of variation of pasture price on the economic value(EV) of feed intake (FI) for the complete breeding cycle in herd 2 (H2).

\section{Conclusões}

A característica taxa de desmama tem grande impacto sobre a rentabilidade, tanto do sistema de produção de ciclo de cria quanto do ciclo completo. A característica rendimento de carcaça foi de grande importância econômica em rebanhos de ciclo completo, uma vez que o aumento do rendimento não ocasionou aumento do custo. Todavia, estudos adicionais devem ser realizados para análise da viabilidade de inclusão desta característica em índices de seleção. Talvez fosse mais realístico utilizar o peso da carcaça, que é determinado pelo peso ao abate e pelo rendimento da carcaça. A característica consumo alimentar, tanto no confinamento quanto na pastagem, representa um relevante impacto econômico e deveria ser considerada em objetivos de seleção para gado de corte. As circunstâncias de produção podem influenciar os valores econômicos das características estudadas, como o peso da vaca à idade adulta, cujo aumento poderia apresentar impactos econômicos positivos ou negativos no lucro anual de rebanhos de corte de acordo com as situações de preços de insumos e de mercado dos produtos. O impacto de mudanças dos preços dos produtos e insumos sobre os valores econômicos das características é maior nos sistemas de produção, nos quais esses preços são mais elevados na situação básica.

\section{Literatura Citada}

AMER, P.R.; KEMP, R.A.; BUCHANAN-SMITH, J.C. et al. A bio economic model for comparing beef cattle genotypes at their optimal economic slaughter end point. Journal of Animal Science, v.72, n.1, p.38-50, 1994

AMER, P.R.; EMMANS, G.C.; SIMM, G. Breeding objectives for beef cattle in Ireland. Livestock Production Science, v.67, n.2, p.223-239, 2001.

ANUALPEC 2003. Anuário da pecuária brasileira. 1.ed. São Paulo: ARGOS Comunicação, 2003. 392p.

BITTENCOURT, T.C.C.; LÔBO, R.B. BEZERRA, L.F. Estimativa de ponderadores econômicos para características de importância econômica em gado de corte, usando equações de lucro. In: CONGRESSO BRASILEIRO DE MEDICINA VETERINÁRIA, 2001, 28., Salvador. Resumos... Brasília: Sociedade Brasileira de Melhoramento Animal, 2001.p.223-223.

BRUMATTI, R.C. Desenvolvimento de um modelo bioeconômico para a determinação de ponderadores econômicos utilizados em índices de seleção em gado de corte. Pirassununga: Universidade de São Paulo, 2002. 113p. Tese (Doutorado em Genética) - Universidade de São Paulo, 2002.

GROEN, A.F.; STEINE, T.; COLLEAU, J.J. et al. et al. Economic values in dairy cattle breeding, with especial reference to functional traits. Report of an EAAP-working group. Livestock Production Science, v.49, p.1-21, 1997.

JORGE JR., J.; CARDOSO, V.L.; ALBUQUERQUE, L.G. Modelo bio econômico para cálculo de custos e receitas em sistemas de produção de gado de corte, visando a obtenção de valores econômicos. Revista Brasileira de Zootecnia, v.5, p.21872196, 2006.

PARNELL, P. Balancing growth, carcass and fertility in your breeding program. Angus Technical Report, n.2, p.1-8, 2000. Disponível em: <http://angusaustralia.com.au> Acesso em: 10/7/2003.

PONZONI, R.W. Genetic improvement of hair sheep in the tropics. Rome: FAO Animal Production Rome and Health, 1992. p.345-445. (Paper, 101).

PONZONI, R.W.; NEWMAN, S. Developing breeding objectives for Australian beef cattle production. Animal Production, n.49, p.35-47, 1989.

SMITH, C.; JAMES, J.W.; BRASCAMP, E.W. On derivation of economics weight in livestock improvement. Animal Production, v.43, p.545, 1986. 